\title{
Molecular pathogenesis of multiple myeloma and its premalignant precursor
}

\author{
W. Michael Kuehl ${ }^{1}$ and P. Leif Bergsagel ${ }^{2}$
}

${ }^{1}$ Genetics Branch, Center for Cancer Research, National Cancer Institute, Bethesda, Maryland, USA ${ }^{2}$ Comprehensive Cancer Center, Mayo Clinic, Scottsdale, Arizona, USA.

\begin{abstract}
Multiple myeloma is a monoclonal tumor of plasma cells, and its development is preceded by a premalignant tumor with which it shares genetic abnormalities, including universal dysregulation of the cyclin $\mathrm{D}$ /retinoblastoma (cyclin $\mathrm{D} / \mathrm{RB}$ ) pathway. A complex interaction with the BM microenvironment, characterized by activation of osteoclasts and suppression of osteoblasts, leads to lytic bone disease. Intratumor genetic heterogeneity, which occurs in addition to intertumor heterogeneity, contributes to the rapid emergence of drug resistance in high-risk disease. Despite recent therapeutic advances, which have doubled the median survival time, myeloma continues to be a mostly incurable disease. Here we review the current understanding of myeloma pathogenesis and insight into new therapeutic strategies provided by animal models and genetic screens.
\end{abstract}

\section{Introduction}

Multiple myeloma (MM) is an age-dependent monoclonal tumor of BM plasma cells (PCs). MM cells are similar to long-lived, postgerminal center (post-GC) PCs, and are characterized by strong BM dependence, extensive somatic hypermutation (SHM) of Ig genes, and absence of IgM expression in all but $1 \%$ of tumors (1). However, MM cells differ from healthy PCs because they retain the potential for a low rate of proliferation ( $1 \%-3 \%$ of cycling cells). MM usually is associated with end-organ damage that can include lytic bone lesions, anemia, immunodeficiency, and decreased renal function (2). It is the second most common hematopoietic malignancy, with an incidence of about 20,000 per year in the United States (3). Despite recent therapeutic advances, MM continues to be a mostly incurable disease, but the median survival has increased from 3 years to over 6 years (4). MM has served as a model for understanding lymphoid tumors because it is characterized by the presence of a premalignant precursor tumor and defined disease stages; researchers have been able to isolate pure tumor cells at all stages. In addition, the study of MM has provided significant knowledge about the critical role of the BM microenvironment in hematopoietic malignancy (5).

\section{Monoclonal gammopathy of undetermined significance is a common premalignant tumor that precedes MM} Monoclonal gammopathy of undetermined significance (MGUS) has a prevalence of $4 \%$ in Caucasians over the age of $50(6,7)$. It can be subclassified as lymphoid (15\%) or PC (85\%) MGUS, which can progress sporadically at average rates of $1 \%$ per year to chronic lymphocytic leukemia, lymphoma, lymphoplasmacytoma, or Waldenstrom's macroglobulinemia, and MM, respectively (8). Lymphoid MGUS and PC MGUS can be distinguished by morphology, but more frequently clinicians use an imperfect method based on the type of monoclonal $\mathrm{Ig}$ (mIg detected in serum or urine: mostly IgM for lymphoid MGUS and mostly non-IgM (including Ig light chain only; ref. 6) for PC MGUS. MGUS is distinguished clinically from MM by having no detectable end-organ damage, a serum mIg of less than $3 \mathrm{~g} / \mathrm{dl}$, and a BM PC content less than $10 \%$ of mononuclear cells (but BM biopsies are not done routinely on these patients) (9).

Conflict of interest: The authors have declared that no conflict of interest exists. Citation for this article: J Clin Invest. 2012;122(10):3456-3463. doi:10.1172/JCI61188.
Although MGUS typically is asymptomatic, some patients develop primary amyloidosis as a result of the accumulation of pathological mIg light chain deposits in various tissues $(2,10)$. Most - if not all - symptomatic MM tumors are preceded by MGUS $(11,12)$. Smoldering MM (SMM) also has no detectable end-organ damage, but differs from MGUS by having a serum $\mathrm{mIg}$ higher than $3 \mathrm{~g} / \mathrm{dl}$ or a BM PC content of more than $10 \%$ and an average rate of progression to symptomatic MM of $10 \%$ per year. Currently there are no tests that measure phenotypic or genotypic markers on tumor cells that predict progression (8). However, two models based on serum and flow cytometric tests stratify patients into groups that progress at yearly rates for MGUS and SMM, respectively, of $0.3 \%$ to $12 \%$ and $0.8 \%$ to $29 \%(8,13-15)$. These models are being used to select high-risk SMM patients for clinical trials $(16,17)$.

\section{An abnormal immunophenotype distinguishes healthy PCs from tumor cells}

Healthy BM PCs are $\mathrm{CD} 38^{+} \mathrm{CD} 138^{+} \mathrm{CD} 19^{+} \mathrm{CD} 45^{+} \mathrm{CD} 56^{-}$. Although MGUS, SMM, and MM tumor cells also are CD $38^{+} \mathrm{CD} 138^{+}, 90 \%$ are $\mathrm{CD} 19^{-}, 99 \%$ are $\mathrm{CD}^{-} 5^{-}$or $\mathrm{CD} 45^{\text {lo }}$, and $70 \%$ are $\mathrm{CD}^{+} 6^{+}(14,18)$. Perhaps the normal cell that is the target of transformation has this abnormal immunophenotype, but no one has identified a normal $\mathrm{BM}$ or primary lymphoid tissue cell with this phenotype. Alternatively, it is possible that transformation activates an epigenetic program that includes the changes in expression of these surface antigens. Despite our inability to explain the abnormal immunophenotype, it provides a useful assay for distinguishing tumor and healthy PCs.

\section{The phenotype of the MM stem/tumor-propagating cell} Unlike CD38- or CD138- cells, $\mathrm{CD} 138^{+}$or $\mathrm{CD} 38^{+} \mathrm{MM}$ cells can proliferate and induce lytic bone lesions when transplanted into ectopic bone in SCID-hu or SCID-rab immunodeficient mouse models (19). This suggests that the tumor-propagating cell has a PC phenotype, with the caveat that it is not possible to serially transplant the cells more than a few cycles. However, recently it was shown that CD138+ but not CD138- cells from two PC leukemia (PCL) tumors could be serially cloned in vitro when cytokines (IL-6 and IGF-1) were included in the media (20). By contrast, others have reported that $\mathrm{CD} 38^{-} \mathrm{CD} 19^{+} \mathrm{CD} 27^{+}$cells, but not $\mathrm{CD} 38^{+}$or $\mathrm{CD} 138^{+}$cells, can form in vitro clones or in vivo tumors in immunodeficient NOD/ 


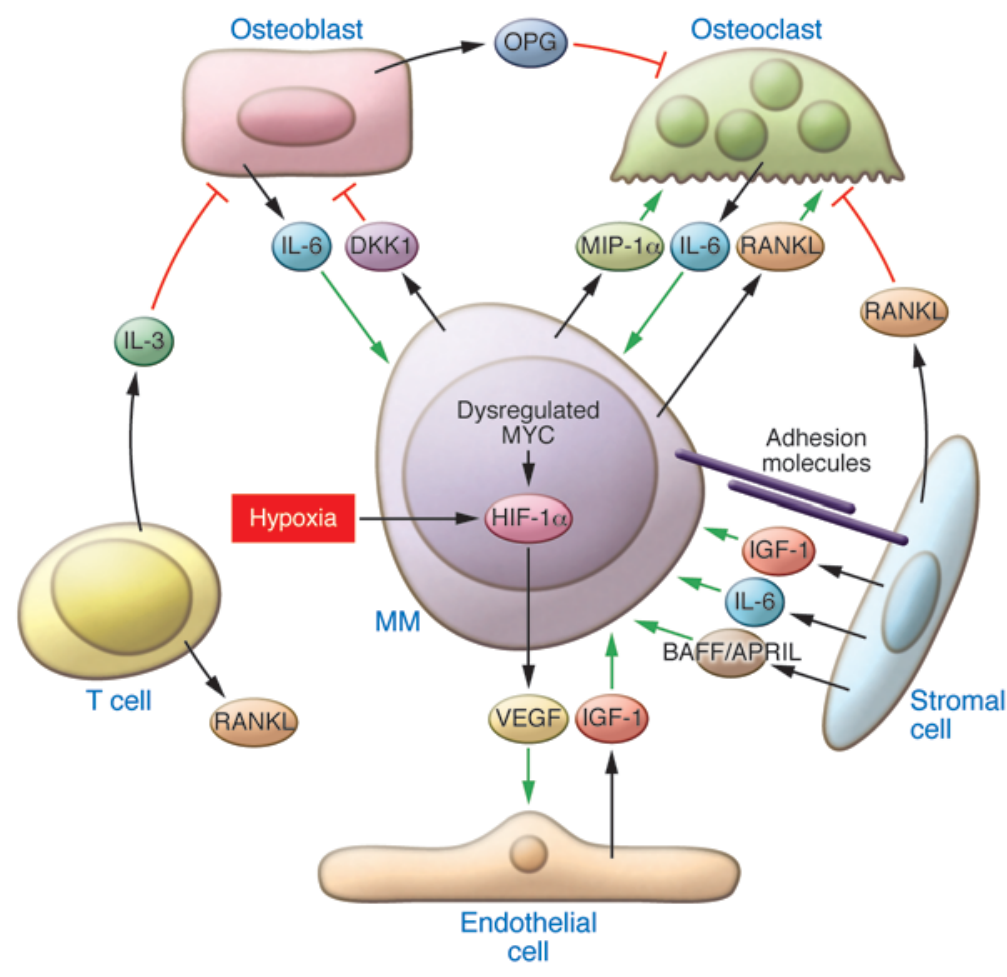

\section{Figure 1}

Interactions of MM tumor cells with the BM microenvironment. Five kinds of cells in the BM microenvironment are depicted, as well as a few of the complex interactions among these cells and MM cells. Some of the critical survival and growth factors, such as IL-6, are made by more than one kind of BM cell. External stimuli, such as hypoxia and internal signals resulting from dysregulated MYC, stimulate HIF- $1 \alpha$ and VEGF secretion, which in turn stimulate endothelial cells to secrete IGF-1. The hallmark uncoupling of bone remodeling is partially explained by an increase in osteoclast activity (mediated by RANKL/RANK interactions, decreased osteoprotegerin (OPG), and increased MIP-1 $\alpha$ ) and a decrease in osteoblast activity (mediated by DKK1 and IL-3). The resultant increase in osteoclast activity stimulates the survival and growth of MM cells, at least partially by increased IL-6. Potential therapeutic agents that directly inhibit some of these interactions include bisphosphonates (which inhibit osteoclast function), anti-RANKL antibody, antiDKK1 antibody, and exogenous OPG.
SCID mice that give rise to $\mathrm{CD} 138^{+}$cells $(21,22)$. This suggests that there is a tumor-propagating cell with a B cell phenotype, although these experiments have not shown that the in vitro clones or in vivo tumors share both the clonotype and the genomic abnormalities that are present in the bulk of the corresponding MM tumor cells (23). We conclude that tumor-propagating cells have a PC phenotype, although it is unknown what fraction of MM tumor cells is capable of replication. However, it remains possible that tumor cells with a $B$ cell phenotype might contribute to progression of MGUS to $\mathrm{MM}$, to tumor propagation and progression, or to relapse after an apparently complete remission.

\section{Symptomatic MM stages}

Progression of symptomatic MM is associated with expanding BM tumor mass and increasingly severe organ impairment or symptoms (2). Despite BM dependence, sometimes the tumor extends to extramedullary locations, such as spleen, liver, and extracellular spaces. Extramedullary MM (EMM) typically is a more aggressive tumor that sometimes is associated with primary or secondary PCL, depending on whether a preceding intramedullary MM was recognized. More than 60 human MM cell lines (HMCLs), which provide a renewable repository of most oncogenic events involved in initiation and progression of the corresponding MM tumor, have been generated, but mostly from EMM tumors $(24,25)$.

\section{A critical but complex role for the BM microenvironment in MM}

Similar to long-lived PCs, MGUS and MM cells are dependent on the BM microenvironment, which includes the extracellular matrix and many kinds of cells, e.g., stromal cells, osteoclasts, osteoblasts, immune cells (T lymphocytes, dendritic cells), other hematopoietic cells and their precursors, and vascular endothelial cells (refs. 1, 26, 27; Figure 1). Reciprocal positive and negative interactions among these cells are mediated by a variety of adhesion molecules, cytokines, and receptors. Additional stimuli such as hypoxia result in activation of HIF- $1 \alpha$ and secretion of VEGF (28). For MM, there are several biological phenomena that are affected by these tumorhost interactions, including homing to BM; spread to secondary BM sites by the bloodstream; generation of many paracrine factors that are involved in the survival, differentiation, and proliferation of tumor cells (most notably IL-6, IGF-1, and APRIL); angiogenesis; osteoclastogenesis; inhibition of osteogenesis; enhanced resistance to chemotherapeutic agents; humoral and cellular immunodeficiency; and anemia. Many of these tumor-host interactions (e.g., homing and differentiation/survival) appear to be qualitatively similar for PC and MM tumor cells, whereas the altered composition of the BM microenvironment represents a pathological response to the MGUS and MM tumor cells. Several therapies (such as immunomodulators and proteasome inhibitors) might target not only the tumor cell but also its interaction with the BM microenvironment. Identifying other therapies directly targeting the microenvironment or its interaction with MM tumor cells is an active area of investigation $(29,30)$.

\section{Seven primary IgH translocations are shared by MM and MGUS tumors}

There are three primary $\operatorname{IgH}$ translocation groups that involve the cyclin d (CCND) family, the MAF family, and Wolf-Hirschhorn syndrome candidate 1/FGFR3 (MMSET/FGFR3) genes (Table 1 and refs. 8, 31). These mostly balanced translocations position an oncogene under control of the $\operatorname{IgH}$ intronic (Emu) and/or $3^{\prime} \operatorname{IgH}$ 
Table 1

Comparison of different molecular classifications in MM

\begin{tabular}{|c|c|c|c|c|c|c|}
\hline Group & TC & Gene & Percent $\mathrm{C}$ & UAMS & HOVON-GMMGA & Comment \\
\hline \multirow[t]{3}{*}{ Cyclin D translocation } & $11 q 13^{B}$ & CCND1 & 15 & CD1, CD2 & CD1, CD2 & Divergent clinical outcomes for CD1 and CD2 \\
\hline & $6 p 21^{B}$ & CCND3 & 2 & $\mathrm{CD} 1, \mathrm{CD} 2$ & $\mathrm{CD} 1, \mathrm{CD} 2$ & Divergent clinical outcomes for CD1 and CD2 \\
\hline & $12 p 13^{B}$ & CCND2 & $<1$ & CD1, CD2 & CD1, CD2 & Divergent clinical outcomes for CD1 and CD2 \\
\hline MMSET translocation & $4 p 16$ & MMSET & 15 & MS & MS & FGFR3 expressed in $75 \%$ of MMs \\
\hline \multirow[t]{3}{*}{ MAF translocation } & $16 q 23$ & MAF & 5 & MF & MF & $\begin{array}{c}\text { Strong transcriptional profile with } \\
\text { expression of ITGB7 }\end{array}$ \\
\hline & $20 q 12$ & $M A F B$ & 2 & MF & MF & $\begin{array}{c}\text { Strong transcriptional profile with } \\
\text { expression of ITGB7 }\end{array}$ \\
\hline & $8 q 24$ & MAFA & $<1$ & MF & MF & $\begin{array}{c}\text { Strong transcriptional profile with } \\
\text { expression of ITGB7 }\end{array}$ \\
\hline \multirow[t]{2}{*}{ HRD } & D1 & CCND1 & 33 & HY & $\begin{array}{l}\text { HY, CD-1, NF-кB, } \\
\text { CTA, PRL3 }\end{array}$ & $\begin{array}{l}\text { NF- } \mathrm{KB} \text { target gene expression } \\
\text { may be ligand dependent or may } \\
\text { result from activating mutations }\end{array}$ \\
\hline & $\mathrm{D} 1+\mathrm{D} 2$ & $\begin{array}{l}\text { CCND1, } \\
\text { CCND2 }\end{array}$ & 7 & PR & PR, CTA & $\begin{array}{l}\mathrm{D} 1+\mathrm{D} 2 \text { might occasionally be a progression } \\
\text { from } \mathrm{D} 1 \text {; PR contains } 5 \%-10 \% \text { of each } \\
\text { TC group, with the exception of D1+D2 and } \\
\text { None (contains }>40 \% \text { of each) }\end{array}$ \\
\hline \multirow[t]{2}{*}{ Other } & None $e^{D}$ & No CCND & 2 & PR & PR CTA & Biallelic RB deletion frequent in None \\
\hline & D2 & CCND2 & 18 & PR LB & LB CTA PRL3 & $\begin{array}{l}\text { PRL3 lacks poor risk features and is } \\
\text { enriched for ISS } 1 \mathrm{E} \text { patients }\end{array}$ \\
\hline
\end{tabular}

AHOVON-GMMG indicates Dutch-Belgian Cooperative Trial group for Hematology-Oncology and German Multiple Myeloma Group. BThe 11q13 and 6p21 are combined into one TC group; the 12p13 is not usually identified and thus is included in the D2 group. PR, proliferation. CPercent refers to the percent of MM patients in each group. DNone refers to a group of patients with no CCND expression. EISS 1, International Staging System (ISS) 1.

$\left(3^{\prime} E\right)$ enhancers. As the breakpoints usually occur near or within IgH switch regions, but sometimes near VDJ sequences, it seems likely that the translocations are related to errors in class switch recombination or SHM, as normal B cells pass through the GC (1). In rare instances, tumors may have translocations involving two of the primary translocation groups, suggesting that there can be some complementation (25).

It is thought that CCND translocations only dysregulate expression of a CCND gene. In contrast, MAF translocations dysregulate expression of a MAF transcription factor that causes increased expression of many genes, including CCND2 and adhesion molecules that are thought to enhance the ability of the tumor cell to interact with the BM microenvironment (32-34). The contributions of the two genes dysregulated by $t(4 ; 14)$ remain controversial. MMSET is a chromatin-remodeling factor that is overexpressed in all tumors with a $\mathrm{t}(4 ; 14)$, whereas about $20 \%$ of tumors lack der(14) and FGFR3 expression. The rare acquisition of FGFR3-activating mutations during progression confirms a role for FGFR3 in MM pathogenesis. Although an activated mutant FGFR3 can be oncogenic, it recently was shown that wild-type FGFR3 (as is found in most $\mathrm{t}[4 ; 14]$ tumors) can also contribute to B cell oncogenesis (35). It remains to be determined whether FGFR3 is critical early in pathogenesis but becomes dispensable during progression of $\mathrm{t}(4 ; 14) \mathrm{MM}$. Preclinical studies suggest that tyrosine kinase inhibitors are active only against $t(4 ; 14)$ HMCL with activating mutations of FGFR3, whereas anti-FGFR3 monoclonal antibodies that inhibit FGFR3 signaling but also elicit antibody-dependent, cell-mediated cytotoxicity are active against HMCLs expressing wild-type FGFR3 $(36,37)$. Despite an apparently indispensable role in $\mathrm{t}(4 ; 14) \mathrm{MM}$, it remains to be determined how MMSET, which sometimes has aminoterminal truncations caused by the translocation, contributes to MM pathogenesis. How- ever, MMSET is a histone methyltransferase for H4K20 and, when overexpressed, results in a global increase in H3K36 methylation and a decrease in H3K27 methylation, which might explain some of the many changes in gene expression associated with $t(4 ; 14)$ tumors $(32,38,39)$. In addition, it was recently determined that MMSET has a role in DNA repair (40). Importantly, loss of MMSET expression alters adhesion, suppresses growth, and results in apoptosis of HMCLs, suggesting that it is an attractive therapeutic target (39).

\section{Chromosome content is associated with different oncogenic pathways}

Nearly half of MGUS and MM tumors are hyperdiploid (HRD), with 48-75 chromosomes (most have 49-56), including extra copies of three or more odd-numbered chromosomes (chromosomes $3,5,7,9,11,15,19$, or 21 ; ref. 31 ). Non-HRD (NHRD) tumors have fewer than 48 and/or more than 75 chromosomes. Strikingly, only about $10 \%$ of HRD tumors have a primary IgH translocation, whereas about 70\% NHRD tumors have an IgH translocation. Tumors with a $t(11 ; 14)$ translocation may represent a distinct category of NHRD tumors, as they often are diploid or pseudodiploid. Curiously, EMM tumors and HMCLs nearly always have a NHRD genotype, suggesting that HRD tumors are more stromal cell dependent than NHRD tumors $(41,42)$. Although it has been proposed that NHRD and HRD tumors represent different pathways of pathogenesis $(31,43)$, the timing, mechanism, and molecular consequences of hyperdiploidy are unknown.

\section{Universal CCND dysregulation in MGUS and MM tumors}

Despite a low proliferation index, there is increased expression of a CCND gene in virtually all MGUS and MM tumors (Figure 2 and refs. 31, 32). Firstly, this is related to direct or indirect dysregula- 


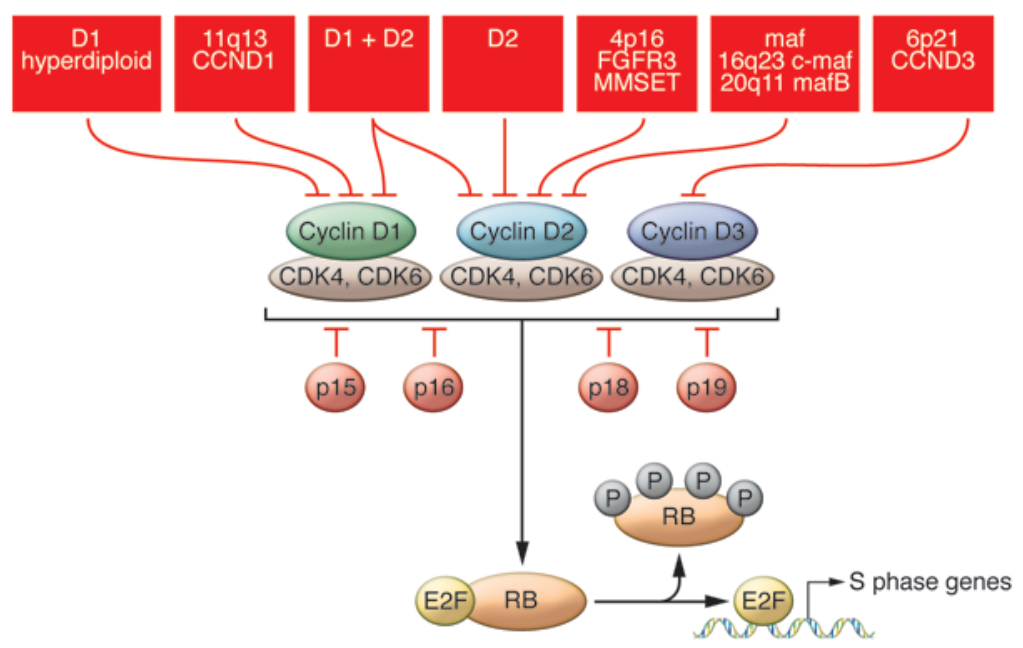

\section{Figure 2}

Early and late disruption of the RB pathway. The early dysregulation of a cyclin $D$ gene provides the basis for the TC classification (see text for details). Yet most MGUSs and most MM tumors are minimally proliferative, perhaps a result of the inhibitory effects of p18INK4c, since p16INK4a usually is not expressed. Increased proliferation at late stages of progress sometimes is associated with inactivation of $\mathrm{p} 18$ or RB1, but most proliferative tumors have a paradoxically high level of $\mathrm{p} 18 \mathrm{INK} 4 \mathrm{c}$ expression and normal levels of RB1. tion, respectively, in tumors with CCND and MAF group translocations. Secondly, although the mechanism is not understood, MMSET/FGFR3 tumors also express moderately increased levels of CCND2. In addition, although normal B cells and PCs do not express CCND1, about two-thirds of MGUS or MM tumors without a primary $\mathrm{IgH}$ translocation (virtually all are HRD) express CCND1, and sometimes CCND2, in a biallelic manner. Notably, an extra copy of chromosome 11 is found mainly in HRD tumors that express CCND1. Most of the remaining tumors (about $40 \%$ of which are HRD) express increased CCND2 compared with normal PCs. Finally, the infrequent $(<5 \%)$ tumors that do not express increased levels of a CCND gene often have inactivated $R B 1$, obviating the need for CCND to stimulate proliferation (31).

\section{Additional oncogenic events in MGUS and MM tumors}

Chromosome 13 deletion. A recent study concludes that chromosome 13 deletion can be an early event in MGUS (e.g., in MAF, MMSET tumors) or a progression event (e.g., in t $[11 ; 14]$ tumors) (44). The pathogenic effect of this chromosome deletion is unknown, though it is possible it may lead to progression due to haploinsufficiency of RB1 (31).

Activating mutations of RAS and BRAF. The prevalence of activating NRAS or KRAS mutations is about $15 \%-18 \%$ each in newly diagnosed and relapsed MM tumors $(31,45)$ but is substantially higher in tumors that express CCND1 compared with tumors that express CCND2. For MGUS tumors, the prevalence of NRAS mutations is $7 \%$, but KRAS mutations have not been described (8). This is consistent with increasing evidence that NRAS and KRAS mutations have overlapping but non-identical effects (46), and with the hypothesis that KRAS mutations provide a molecular mark of the transition of MGUS to MM $(23,47)$. MM tumors depend on the continued expression of activated but not wild-type RAS (48). Recently, BRAF mutations were described in $4 \%$ of MM tumors, suggesting a possible role for BRAF inhibitors in these cases (49).

$M Y C$ dysregulation. There is increased expression of $\mathrm{c}-M Y C$ in most newly diagnosed MM tumors compared with MGUS tumors (50). Recently it was shown that sporadic activation of a MYC transgene in GC B cells in an MGUS-prone mouse strain leads to the universal development of MM tumors $(51,52)$. Hence, increased MYC expression seems to be responsible for progression from MGUS to MM. Complex translocations involving $M Y C$ (c-MYC $>>\mathrm{N}-M Y C>\mathrm{L}-M Y C)$ appear to be secondary progression events that often do not involve Ig loci (53). They are rare or absent in MGUS but occur in $15 \%$ of newly diagnosed tumors, $50 \%$ of advanced tumors, and $90 \%$ of HMCLs $(25,54)$. A recent report suggested that a small molecule inhibitor of BRD4 can inhibit MYC RNA expression in MM (55).

Mutations that activate the NF- $\mathrm{\kappa} B$ pathway. Extrinsic ligands (APRIL and BAFF) produced by BM stromal cells provide critical survival signals to long-lived PCs by stimulating TACI, BCMA, and BAFF receptors to activate the NF- $\kappa B$ pathways (56). Most MGUS and MM tumors highly express NF-кB target genes, suggesting a continued role of extrinsic signaling in PC tumors $(57,58)$. Activating mutations in positive regulators and inactivating mutations in negative regulators of the NF- $\kappa \mathrm{B}$ pathway have been identified in at least $20 \%$ of untreated MM tumors and approximately $50 \%$ of HMCLs, rendering the cells less dependent on ligand-mediated NF-кB activation (49). Small molecules that inhibit extrinsic signaling (including TACI.Fc, IKK $\beta$, and NIK [MAP3K14]) are being developed as potential therapeutic agents $(59,60)$. There is also some evidence suggesting that cells addicted to constitutive NF- $\kappa \mathrm{B}$ activation may be particularly sensitive to proteasome inhibition (58).

Chromosome 17p loss and abnormalities of TP53. Deletions that include the TP53 locus occur in approximately $10 \%$ of untreated MM tumors, and the prevalence increases with disease stage (31, 42). TP53 mutations were present in $37 \%$ of untreated MM tumors with del17p, but not in patients without del17p (61). It remains to be determined whether the poor prognosis associated with monoallelic del17p but no TP53 mutation is due to haploinsufficiency or to predisposition to complete inactivation of TP53. Recently, decreased expression of microRNAs miR199, miR192, and miR215 in MM was reported to increase MDM2, an inhibitor of TP53 (62).

Gain of chromosome 1q and loss of chromosome 1p. These genomic events frequently occur together in $\mathrm{MM}$, and each is associated with a poor prognosis $(31,63)$. The relevant genes on $1 \mathrm{q}$ are unclear at this time. By contrast, there are potential targets on two regions of $1 \mathrm{p}$ that are associated with a poor prognosis: $C D K N 2 C(\mathrm{p} 18 \mathrm{INK} 4 \mathrm{c})$ at $1 \mathrm{p} 32.3$ and FAM46C at 1p12 $(64,65)$. Homozygous deletion of CDKN2C, which is present in about $30 \%$ of HMCL and about $5 \%$ of untreated MM tumors, is associated with increased proliferation and a poor prognosis, whereas monoallelic deletion is not. Mutations of FAM46C often with hemizygous deletion - were identified in 3.4\% and $13 \%$ of MM tumors in two studies, and in $25 \%$ of 16 HMCL $(49,64)$. 


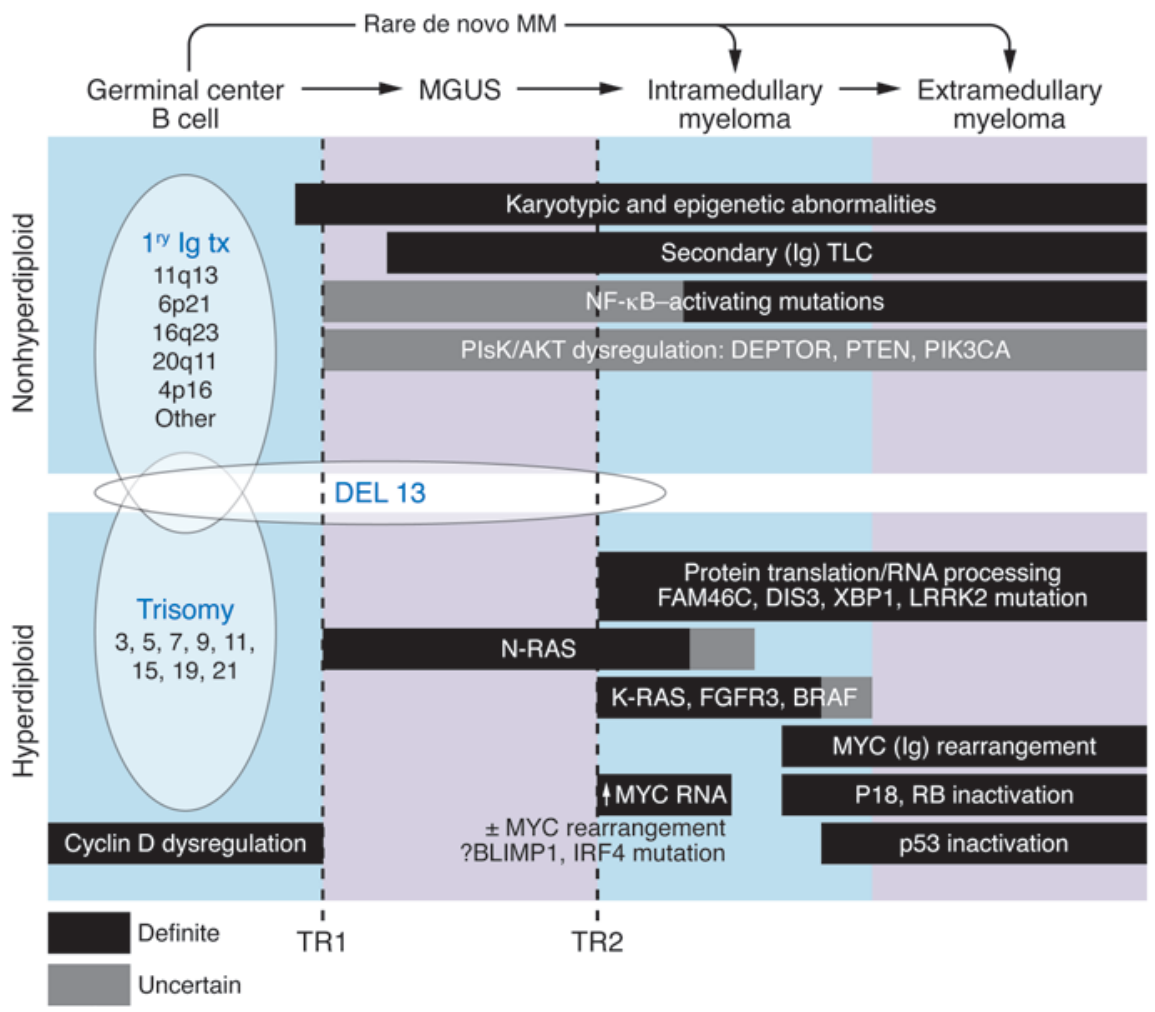

Figure 3

Model for molecular pathogenesis of MGUS and MM. The initial transition (TR1) to a recognizable tumor involves two mostly non-overlapping pathways (IgH translocations versus multiple trisomies) that include primary events associated with dysregulated cyclin D expression in MGUS and MM. The transition from MGUS to MM (TR2) is associated with increased MYC expression and sometimes with activating mutations of K-RAS or chromosome 13 deletion. Early and late progression events for symptomatic MM tumors are shown.

Other pathogenic events. Secondary Ig translocations, including most IgK and IgL translocations and IgH translocations not involving one of the seven primary partners, can occur at all stages of disease, and with a similar frequency in HRD and NHRD tumors, but apart from MYC, few partner loci have been identified (25). Other genomic rearrangements are frequent, but only a few specific target genes have been identified $(63,66,67)$. Changes in DNA methylation are frequent, with one study suggesting that a marked increase in hypomethylation is associated with the MGUS-to-MM transition (68), whereas a second study suggests only a small increase in hypomethylation for MM compared with MGUS (69). Mutations in seven genes regulating RNA metabolism, protein translation, and homeostasis were identified in 16 of 38 patients (49). In addition to previous studies implicating roles for MMSET and KDM6A (UTX), genomic sequencing studies found that other histonemodifying enzymes are frequent targets of mutation, although the epigenetic consequences are unknown (49). Similarly, changes in microRNA expression at different stages have been identified, but more extensive studies are needed $(62,70)$.

\section{Model for molecular pathogenesis of MGUS and MM}

The pathogenesis of MGUS and MM can be considered as occurring in three phases (Figure 3 and refs. 8, 34). Early, partially overlapping genetic events common to MGUS and MM include at a minimum primary IgH translocations, hyperdiploidy, and del13 that lead directly or indirectly to dysregulation of a CCND gene.
Second, the transition from MGUS to MM is associated with increased MYC expression and sometimes KRAS mutations, but can also include del13 in t $(11 ; 14)$ tumors. Finally, further progression of the MM tumor seems to be associated with other events. For example, increased proliferation and genomic instability, and decreased dependence on the BM microenvironment, sometimes including extramedullary spread of disease, can be associated with late MYC rearrangements that often involve an Ig locus, activating mutations of the NF-кB pathway, deletion or mutation of TP53, and inactivation of $p 18 I N K 4 c$ or $R B 1$.

\section{Clinical implications of molecular classifications}

The presence of primary IgH translocations and the universal overexpression of CCND genes led to the development of the translocations and cyclin D (TC) classification that is focused mainly on early events common to MGUS and MM, and therefore is applicable to the classification of both MGUS and MM tumors (Table 1). Unsupervised analyses of microarray gene expression profiling (GEP) have identified additional MM tumor groups with shared patterns of gene expression $(71,72)$ that highlight other important secondary events that can occur in each subtype of MM: proliferation and expression of NF- $\mathrm{KB}$ target genes, cancer-testis antigens (CTAs), and the phosphatase PTP4A3/PRL3. The University of Arkansas for Medical Science (UAMS) CD1 and CD2 classification groups represent subgroups of patients with $t(11 ; 14)$ and $t(6 ; 14)$ tumors, with the former characterized by arginosuccinate synthetase 1 expression 


\section{Table 2}

Survival of high-risk genetic subgroups in randomized, controlled clinical trials in patients with untreated MM

\begin{tabular}{|c|c|c|c|c|c|c|c|}
\hline Genetic lesion ${ }^{A}$ & Arm 1 n/Arm 2 n & Endpoint & $\operatorname{Arm} 1^{B}$ & $\operatorname{Arm} 2^{B}$ & Arm 1 OSC & Arm 2 OSC & Reference \\
\hline $\mathrm{t}(4 ; 14)$ & $33 / 31$ & $3-y r$ OS & V-A-D/HDM/Thal & Bor-A-D/HDM/Bor & $44 \%$ & $66 \%$ & 76 \\
\hline $\mathrm{t}(4 ; 14)$ & $98 / 106$ & 4-yr OS & $V-A-D$ & Bor-D & $32 \%$ & $63 \%$ & 74 \\
\hline $\mathrm{t}(4 ; 14)$ & $21 / 23$ & $2-y r$ OS & Thal-TT2 & Placebo-TT2 & $67 \%$ & $87 \%$ & 75 \\
\hline $\mathrm{t}(4 ; 14)$ & $21 / 29$ & $2-y r$ OS & Thal-TT2 & Bor-TT3 & $67 \%$ & $97 \%$ & 75 \\
\hline del17p & $39 / 19$ & $3-y r$ OS & V-A-D/HDM/Thal & Bor-A-D/HDM/Bor & $17 \%$ & $69 \%$ & 76 \\
\hline del17p & $119 / 54$ & 4-yr OS & V-A-D & Bor-D & $36 \%$ & $50 \%$ & 74 \\
\hline NHRD & $92^{D}$ & 3-yr OS & Thal-D-Bor & Mel-P-Bor & $53 \%$ & $72 \%$ & 79 \\
\hline Unfav. FISH & $152 / 141$ & $3-y r$ OS & Thal-D-Cyclo & V-A-D-Cyclo & $58 \%$ & $56 \%$ & 80 \\
\hline Unfav. FISH & $96 / 90$ & $3-y r$ OS & Thal-D-Cyclo & Placebo-P-Mel & $34 \%$ & $26 \%$ & 81 \\
\hline Unfav. FISH & $99 / 98$ & $3-y r$ OS & Thal maint & Placebo maint & $45 \%$ & $69 \%$ & 82 \\
\hline
\end{tabular}

ANHRD status determined by flow cytometry. Unfavorable (Unfav.) FISH includes any of the following: $t(4 ; 14), t(14 ; 16)$, $t(14 ; 20)$, gain(1q), del(1p32), or del(17p). BRandomized drugs in each arm are shown in bold. ${ }^{C}$ OSs that are significantly different from control are shown in bold. A, adriamycin; Bor, bortezomib; Cyclo, cyclophosphamide; D, dexamethasone; HDM, high-dose intravenous melphalan; maint, maintenance (implies combination and/or sequential therapies); Mel, low-dose oral melphalan; P, prednisone; Thal, thalidomide; TT2, total therapy 2 (an intense multi-drug combination induction, tandem transplant,

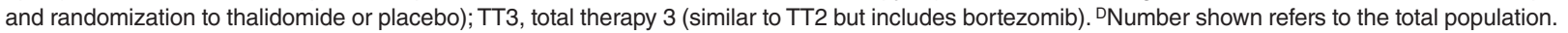

and the later by expression of $\mathrm{B}$ cell antigens (CD20, VPREB, CD79A). Interestingly, CD1 and CD2 groups identify patients with markedly different clinical outcomes. Of the various genetic events in $\mathrm{MM}$, the one most important clinically is the $\mathrm{t}(4 ; 14)$ chromosome translocation. It is associated with a poor prognosis in patients treated with alkylating agents, immunomodulatory drugs (IMiDs), and bortezomib. However, there is a clear survival advantage to the upfront use of bortezomib versus control in these patients (73-76), with a suggestion that prolonged use totally overcomes the adverse prognosis. Numerous randomized, controlled clinical trials of IMiDs in the treatment of thousands of MM patients have been performed, with several studies showing improvements in overall survival (OS) for the cohort receiving IMiDs relative to the control group. Unfortunately, we do not know which molecular subgroups received the maximum benefit from IMiDs versus those that received no benefit. From these studies there are a few reports of the effects of IMiDs on the survival of a molecular subgroup (Table 2). In summary, it appears that thalidomide is no better and often is even worse than placebo in patients with high-risk genetic features (e.g., t [4;14], t[14;16], and del17p). Further studies are urgently required to define the utility and safety of IMiDs in the various molecular subtypes of MM.

The MF molecular subgroup, $t(14 ; 16)$, and $t(14 ; 20)$ tumors have each individually been associated with a poor prognosis. In addition, del17p is universally associated with poor prognosis. Finally, patients defined as high risk by a GEP index of proliferation or other GEP-defined risk scores (which all appear to discriminate prognosis equally in an independent dataset) do poorly (77). Unlike the $t(4 ; 14)$ group, for these latter subgroups neither bortezomib nor any other intervention has been shown to offer a survival advantage, although the data are unfortunately very limited. These patients should be considered for clinical trials exploring innovative approaches. Recently a high level of intraclonal tumor heterogeneity has been described in patients with high-risk MM (67), associated in one case with alternating clonal dominance under therapeutic selective pressure; these observations have important clinical implications. The findings suggest a competition between subclones for limited resources and raise the possibility that early, suboptimal treatment may eradicate the "good," drugsensitive clone, making room for the "bad," drug-resistant clone to expand. They support the use of aggressive multidrug combination approaches for high-risk disease with unstable genomes and clonal heterogeneity and sequential one- or two-drug approaches for lowrisk disease with stable genomes and lacking clonal heterogeneity.

\section{Challenges for the future}

Despite marked progress in understanding the molecular pathogenesis of MM, many important questions remain unanswered. What are the phenotypic and genotypic markers that distinguish MGUS, SMM, and MM, and can they be used to predict progression or suggest therapeutic strategies that will prevent or delay progression? What is the basis for the immunophenotype that distinguishes healthy PCs from tumor PCs? What are the molecular mechanisms and oncogenic consequences of hyperdiploidy? Will studies on cell lines and current animal models provide an adequate way of determining the biological effects - and value as therapeutic targets - of known genetic and epigenetic abnormalities? How can we achieve a more profound understanding of the critical interactions of the BM microenvironment with healthy and tumor PCs? Finally, do current therapeutic regimens show differential activity for tumors with different genetic and phenotypic abnormalities?

Developing new therapeutic strategies is critical. One popular notion is to convert MM to a premalignant MGUS tumor if a complete elimination of MM tumor cells cannot be achieved. However, it will be a challenge to figure out how to effectively monitor this outcome, given our poor understanding of intrinsic differences between MM and MGUS tumors. Simultaneous therapeutic targeting of several genetic and/or epigenetic abnormalities present in individual MM tumors is another attractive concept. But it remains unclear whether initiating or early oncogenic abnormalities are more effective targets than secondary oncogenic abnormalities. Alternatively, it may be possible to target addiction of the tumor cell to the PC phenotype, as illustrated by the dependence of survival of MM cell lines on expression of IRF4 (78). Finally, given that the BM seems to be altered during tumor progression, the possibility of targeting the microenvironment and/or its interaction with tumor cells (including possible enhancement of immune responses) seems attractive, but currently our limited understanding of these interactions hampers this approach. However, the development of an orthotopic, immunocompetent, genetically engineered murine model is a crucial step forward $(51,52)$. 


\section{Acknowledgments}

We thank Marta Chesi for many fruitful discussions. This work was supported by the NIH grants CA100707, CA136671, CA133966, and AG020686 (to P.L. Bergsagel).
Address correspondence to: P. Leif Bergsagel, Mayo Clinic College of Medicine, 13208 E. Shea Blvd, C-007, Scottsdale, Arizona 10021, USA. Phone: 480.301.4704; Fax: 480.301.7017; E-mail: bergsagel. leif@mayo.edu.
1. Kuehl WM, Bergsagel PL. Multiple myeloma: evolving genetic events and host interactions. Nat Rev Cancer. 2002;2(3):175-187.

2. Malpas JS, Bergsagel DE, Kyle R, Anderson K. Multiple Myeloma: Biology and Management. Oxford, United Kingdom: Oxford University Press; 2004.

3. Siegel R, Naishadham D, Jemal A. Cancer statistics, 2012. CA Cancer J Clin. 2012;62(1):10-29.

4. Mahindra A, Laubach J, Raje N, Munshi N, Richardson PG, Anderson K. Latest advances and current challenges in the treatment of multiple myeloma. Nat Rev Clin Oncol. 2012;9(3):135-143.

5. Palumbo A, Anderson K. Multiple myeloma. NEngl JMed. 2011;364(11):1046-1060.

6. Dispenzieri A, et al. Prevalence and risk of progression of light-chain monoclonal gammopathy of undetermined significance: a retrospective population-based cohort study. Lancet. 2010; 375(9727):1721-1728.

7. Kyle RA, et al. Prevalence of monoclonal gammopathy of undetermined significance. $N$ Engl J Med 2006;354(13):1362-1369.

8. Zingone A, Kuehl WM. Pathogenesis of monoclonal gammopathy of undetermined significance and progression to multiple myeloma. Semin Hematol. 2011;48(1):4-12

9. Kyle RA, et al. Monoclonal gammopathy of undetermined significance (MGUS) and smoldering (asymptomatic) multiple myeloma: IMWG consensus perspectives risk factors for progression and guidelines for monitoring and management. Leukemia. 2010;24(6):1121-1127.

10. Bochtler T, et al. Evaluation of the cytogenetic aberration pattern in amyloid light chain amyloidosis as compared with monoclonal gammopathy of undetermined significance reveals common pathways of karyotypic instability. Blood. 2008;111(9):4700-4705.

11. Landgren $\mathrm{O}$, et al. Monoclonal gammopathy of undetermined significance (MGUS) consistently precedes multiple myeloma: a prospective study. Blood. 2009;113(22):5412-5417.

12. Weiss BM, Abadie J, Verma P, Howard RS, Kuehl WM. A monoclonal gammopathy precedes multiple myeloma in most patients. Blood. 2009;113(22):5418-5422.

13. Dispenzieri A, et al. Immunoglobulin free light chain ratio is an independent risk factor for progression of smoldering (asymptomatic) multiple myeloma. Blood. 2008;111(2):785-789.

14. Perez-Persona E, et al. New criteria to identify risk of progression in monoclonal gammopathy of uncertain significance and smoldering multiple myeloma based on multiparameter flow cytometry analysis of bone marrow plasma cells. Blood. 2007;110(7):2586-2592.

15. Rajkumar SV, et al. Serum free light chain ratio is an independent risk factor for progression in monoclonal gammopathy of undetermined significance. Blood. 2005;106(3):812-817.

16. Korde N, et al. 2944 A Phase II Trial of IPH2101 (anti-KIR mAb) in Smoldering Multiple Myeloma ASH Annual Meeting Abstracts. 2011;118:2944.

17. Mateos MV, et al. Smoldering Multiple Myeloma (SMM) at high-risk of progression to symptomatic disease: A Phase III, randomized, multicenter trial based on Lenalidomide-Dexamethasone (LenDex) as induction therapy followed by maintenance therapy with len alone vs no treatment. $A S H$ Annual Meeting Abstracts. 2011;118:991.

18. Raja KR, Kovarova L, Hajek R. Review of phenotypic markers used in flow cytometric analysis of MGUS and MM, and applicability of flow cytometry in other plasma cell disorders. Br J Haematol. 2010;149(3):334-351.

19. Yata K, Yaccoby S. The SCID-rab model: a novel in vivo system for primary human myeloma demonstrating growth of CD138-expressing malignant cells. Leukemia. 2004;18(11):1891-1897.

20. Chiron $\mathrm{D}$, et al. The peripheral CD138(+) population but not the CD138(-) population contains myeloma clonogenic cells in plasma cell leukaemia patients. Br J Haematol. 2012;156(5):679-683.

21. Kukreja A, et al. Enhancement of clonogenicity of human multiple myeloma by dendritic cells. J Exp Med. 2006;203(8):1859-1865.

22. Matsui W, et al. Clonogenic multiple myeloma progenitors, stem cell properties, and drug resistance. Cancer Res. 2008;68(1):190-197.

23. Rasmussen T. Identification of translocation products but not K-RAS mutations in memory B cells from patients with multiple myeloma. Haematologica. 2010;95(10):1730-1737.

24. Drexler HG, Matsuo Y. Malignant hematopoietic cell lines: in vitro models for the study of multiple myeloma and plasma cell leukemia. Leuk Res. 2000;24(8):681-703.

25. Gabrea A, et al. Secondary genomic rearrangements involving immunoglobulin or MYC loci show similar prevalences in hyperdiploid and nonhyperdiploid myeloma tumors. Genes Chromosomes Cancer. 2008;47(7):573-590.

26. Anderson KC, Carrasco RD. Pathogenesis of myeloma. Annu Rev Pathol. 2011;6:249-274.

27. Esteve FR, Roodman GD. Pathophysiology of myeloma bone disease. Best Pract Res Clin Haematol. 2007;20(4):613-624.

28. Hu J, et al. Targeting the multiple myeloma hypoxic niche with TH-302, a hypoxia-activated prodrug. Blood. 2010;116(9):1524-1527.

29. Hideshima T, Mitsiades C, Tonon G, Richardson PG, Anderson KC. Understanding multiple myeloma pathogenesis in the bone marrow to identify new therapeutic targets. Nat Rev Cancer. 2007; 7(8):585-598

30. Roodman GD. Targeting the bone microenvironment in multiple myeloma.J Bone Miner Metab. 2010; 28(3):244-250.

31. Fonseca $\mathrm{R}$, et al. International Myeloma Working Group molecular classification of multiple myeloma: spotlight review. Leukemia. 2009;23(12):2210-2221.

32. Bergsagel PL, Kuehl WM, Zhan F, Sawyer J, Barlogie B, Shaughnessy J Jr. Cyclin D dysregulation: an early and unifying pathogenic event in multiple myeloma. Blood. 2005;106(1):296-303.

33. Hurt EM, et al. Overexpression of c-maf is a frequent oncogenic event in multiple myeloma that promotes proliferation and pathological interactions with bone marrow stroma. Cancer Cell. 2004;5(2):191-199.

34. Bergsagel PL, Kuehl WM. Molecular pathogenesis and a consequent classification of multiple myeloma. J Clin Oncol. 2005;23(26):6333-6338.

35. Zingone A, et al. Ectopic expression of wild-type FGFR3 cooperates with MYC to accelerate development of B-cell lineage neoplasms. Leukemia. 2010; 24(6):1171-1178.

36. Qing J, et al. Antibody-based targeting of FGFR3 in bladder carcinoma and $t(4 ; 14)$-positive multiple myeloma in mice. J Clin Invest. 2009; 119(5):1216-1229

37. Trudel S. CHIR-258, a novel, multitargeted tyrosine kinase inhibitor for the potential treatment of $\mathrm{t}(4 ; 14)$ multiple myeloma. Blood. 2005; 105(7):2941-2948.
38. Marango J, et al. The MMSET protein is a histone methyltransferase with characteristics of a transcriptional corepressor. Blood. 2008;111(6):3145-3154.

39. Martinez-Garcia E, et al. The MMSET histone methyl transferase switches global histone methylation and alters gene expression in $\mathrm{t}(4 ; 14)$ multiple myeloma cells. Blood. 2011;117(1):211-220.

40. Pei $\mathrm{H}$, et al. MMSET regulates histone H4K20 methylation and 53BP1 accumulation at DNA damage sites. Nature. 2011;470(7332):124-128.

41. Avet-Loiseau H, et al. Cytogenetic, interphase, and multicolor fluorescence in situ hybridization analyses in primary plasma cell leukemia: a study of 40 patients at diagnosis, on behalf of the Intergroupe Francophone du Myelome and the Groupe Francais de Cytogenetique Hematologique. Blood. 2001;97(3):822-825.

42. Tiedemann RE, et al. Genetic aberrations and survival in plasma cell leukemia. Lenkemia. 2008; 22(5):1044-1052.

43. Smadja NV, et al. Chromosomal analysis in multiple myeloma: cytogenetic evidence of two different diseases. Leukemia. 1998;12(6):960-969.

44. Chiecchio L, et al. Timing of acquisition of deletion 13 in plasma cell dyscrasias is dependent on genetic context. Haematologica. 2009;94(12):1708-1713.

45. Chng WJ, et al. Clinical and biological significance of RAS mutations in multiple myeloma. Leukemia. 2008;22(12):2280-2284.

46. Haigis KM, et al. Differential effects of oncogenic $\mathrm{K}$-Ras and $\mathrm{N}$-Ras on proliferation, differentiation and tumor progression in the colon. Nat Genet. 2008; 40(5):600-608.

47. Rasmussen T, Kuehl M, Lodahl M, Johnsen HE, Dahl IMS. Possible roles for activating RAS mutations in the MGUS to MM transition and in the intramedullary to extramedullary transition in some plasma cell tumors. Blood. 2005;105(1):317-323.

48. Steinbrunn T, et al. Mutated RAS and constitutively activated Akt delineate distinct oncogenic pathways, which independently contribute to multiple myeloma cell survival. Blood. 2011;117(6):1998-2004.

49. Chapman MA, et al. Initial genome sequencing and analysis of multiple myeloma. Nature. 2011; 471(7339):467-472.

50. Chng WJ, et al. Clinical and biological implications of MYC activation: a common difference between MGUS and newly diagnosed multiple myeloma. Leukemia. 2011;25(6):1026-1035.

51. Chesi M, et al. AID-dependent activation of a MYC transgene induces multiple myeloma in a conditional mouse model of post-germinal center malignancies. Cancer Cell. 2008;13(2):167-180.

52. Chesi $\mathrm{M}$, et al. Drug response in a genetically engineered mouse model of multiple myeloma is predictive of clinical efficacy. Blood. 2012;120(2):376-385.

53. Dib A, Gabrea A, Glebov OK, Bergsagel PL, Kuehl WM. Characterization of MYC translocations in multiple myeloma cell lines. J Natl Cancer Inst Monogr. 2008;(39):25-31.

54. Avet-Loiseau H, Gerson F, Magrangeas F, Minvielle S, Harousseau JL, Bataille R. Rearrangements of the c-myc oncogene are present in $15 \%$ of primary human multiple myeloma tumors. Blood. 2001;98(10):3082-3086.

55. Delmore JE, et al. BET bromodomain inhibition as a therapeutic strategy to target c-Myc. Cell. 2011; 146(6):904-917.

56. Elgueta R, de Vries VC, Noelle RJ. The immortality of humoral immunity. Immunol Rev. 2010;236:139-150.

57. Annunziata CM, et al. Frequent engagement of the classical and alternative NF-kappaB pathways by 
diverse genetic abnormalities in multiple myeloma. Cancer Cell. 2007;12(2):115-130.

58. Keats JJ, et al. Promiscuous mutations activate the noncanonical NF-kappaB pathway in multiple myeloma. Cancer Cell. 2007;12(2):131-144.

59. Demchenko YN, Glebov OK, Zingone A, Keats JJ, Bergsagel PL, Kuehl WM. Classical and/or alternative NF-kappaB pathway activation in multiple myeloma. Blood. 2010;115(17):3541-3552.

60. Rossi JF, et al. Atacicept in relapsed/refractory multiple myeloma or active Waldenström's macroglobulinemia: a phase I study. Br J Cancer. 2009; 101(7):1051-1058.

61. Lode L, et al. Mutations in TP53 are exclusively associated with del(17p) in multiple myeloma. Haematologica. 2010;95(11):1973-1976.

62. Pichiorri F, et al. Downregulation of p53-inducible microRNAs 192, 194, and 215 impairs the p53/ MDM2 autoregulatory loop in multiple myeloma development. Cancer Cell. 2010;18(4):367-381.

63. Walker BA, et al. A compendium of myelomaassociated chromosomal copy number abnormalities and their prognostic value. Blood. 2010; 116(15):e56-e65.

64. Boyd KD, et al. Mapping of chromosome 1p deletions in myeloma identifies FAM46C at 1p12 and $\mathrm{CDKN} 2 \mathrm{C}$ at $1 \mathrm{p} 32.3$ as being genes in regions associated with adverse survival. Clin Cancer Res. 2011; 17(24):7776-7784

65. Dib A, et al. Paradoxical expression of INK4c in proliferative multiple myeloma tumors: bi-allelic deletion vs increased expression. Cell Div. 2006;1:23.
66. Avet-Loiseau H, et al. Prognostic significance of copy-number alterations in multiple myeloma. J Clin Oncol. 2009;27(27):4585-4590.

67. Keats JJ, et al. Clonal competition with alternating dominance in multiple myeloma. Blood. 2012;120(5):1067-1076.

68. Walker BA, et al. Aberrant global methylation patterns affect the molecular pathogenesis and prognosis of multiple myeloma. Blood. 2011;117(2):553-562.

69. Salhia B, Baker A, Ahmann G, Auclair D, Fonseca $\mathrm{R}$, Carpten J. DNA methylation analysis determines the high frequency of genic hypomethylation and low frequency of hypermethylation events in plasma cell tumors. Cancer Res. 2010;70(17):6934-6944.

70. Pichiorri F, et al. MicroRNAs regulate critical genes associated with multiple myeloma pathogenesis. Proc Natl Acad Sci U S A. 2008;105(35):12885-12890.

71. Zhan F, et al. The molecular classification of multiple myeloma. Blood. 2006;108(6):2020-2028.

72. Broyl A, et al. Gene expression profiling for molecular classification of multiple myeloma in newly diagnosed patients. Blood. 2010;116(14):2543-2553.

73. San-Miguel JF, et al. Bortezomib plus melphalan and prednisone for initial treatment of multiple myeloma. N Engl J Med. 2008;359(9):906-917.

74. Avet-Loiseau H, et al. Bortezomib plus dexamethasone induction improves outcome of patients with $\mathrm{t}(4 ; 14)$ myeloma but not outcome of patients with del(17p). J Clin Oncol. 2010;28(30):4630-4634.

75. Pineda-Roman M. Sustained complete remissions in multiple myeloma linked to bortezomib in total therapy 3: comparison with total therapy $2 . \mathrm{BrJ}$
Haematol. 2008;140(6):625-634.

76. Neben K, et al. Administration of bortezomib before and after autologous stem-cell transplantation improves outcome in multiple myeloma patients with deletion 17p. Blood. 2012;119(4):940-948.

77. Hose D, et al. Proliferation is a central independent prognostic factor and target for personalized and risk-adapted treatment in multiple myeloma. Haematologica. 2011;96(1):87-95.

78. Shaffer AL, et al. IRF4 addiction in multiple myeloma. Nature. 2008;454(7201):226-231.

79. Mateos M-V, et al. Bortezomib, melphalan, and prednisone versus bortezomib, thalidomide, and prednisone as induction therapy followed by maintenance treatment with bortezomib and thalidomide versus bortezomib and prednisone in elderly patients with untreated multiple myeloma: a randomised trial. Lancet Oncol. 2010;11(10):934-941.

80. Morgan GJ, et al. Cyclophosphamide, thalidomide, and dexamethasone as induction therapy for newly diagnosed multiple myeloma patients destined for autologous stem-cell transplantation: MRC Myeloma IX randomized trial results. Haematologica. 2012;97(3):442-450.

81. Morgan GJ, et al. Cyclophosphamide, thalidomide, and dexamethasone (CTD) as initial therapy for patients with multiple myeloma unsuitable for autologous transplantation. Blood. 2011;118(5):1231-1238.

82. Morgan GJ, et al. The role of maintenance thalidomide therapy in multiple myeloma: MRC Myeloma IX results and meta-analysis. Blood. 2012;119(1):7-15. 